\title{
Targeted delivery of propionate to the colon stimulates the release of anorectic gut hormones and suppresses appetite in humans
}

\author{
E. S. Chambers ${ }^{1}$, A. Viardot ${ }^{1}$, A. Psichas ${ }^{1}$, D. J. Morrison ${ }^{2}$, K. G. Murphy ${ }^{3}$, \\ S. E. K. Zac-Varghese ${ }^{3}$, K. MacDougall ${ }^{4}$, T. Preston ${ }^{2}$, M. C. Tedford ${ }^{4}$, G. Finlayson ${ }^{5}$, \\ J. E. Blundell ${ }^{5}$, W. S. Dhillo ${ }^{3}$, S. R. Bloom ${ }^{3}$, W. G. Morley ${ }^{6}$, S. Clegg $^{6}$ and G. Frost ${ }^{1}$ \\ ${ }^{1}$ Nutrition and Dietetic Research Group, ${ }^{2}$ Scottish Universities Environmental Research Centre, University of Glasgow, \\ G75 0QF, UK, ${ }^{3}$ Section of Investigative Medicine, Imperial College London, W12 0NN, UK, ${ }^{4}$ School of Science, \\ University of the West of Scotland, Paisley PAI 2BE, UK, ${ }^{5}$ Institute of Psychological Sciences, University of Leeds, \\ LS2 9JT, UK and ${ }^{6}$ Leatherhead Food Research, Leatherhead KT22 7RY, UK
}

Recent evidence suggests that short chain fatty acids (SCFA) trigger the release of the anorectic gut hormones peptide YY (PYY) and glucagon like peptide-1 (GLP-1) by stimulating the free fatty acid receptors (FFAR) 2 and 3 on enteroendocrine L cells ${ }^{(1)}$. Of the SCFA produced by colonic fermentation of available carbohydrates, propionate has been shown to have the highest affinity for FFAR2 ${ }^{(2)}$. We hypothesised that increasing colonic propionate would stimulate gut hormone secretion and reduce energy intake. To elevate colonic propionate levels we have developed a novel propionate ester molecule whereby propionate is chemically bound by an ester bond to inulin. The majority of the bound propionate should only be released when the inulin polymer is fermented by the colonic microbiota, thus providing targeted colonic delivery.

To assess the site and extent of propionate release, 9 volunteers consumed a standardised breakfast containing $100 \mathrm{mg}$ of ${ }^{13} \mathrm{C}$ labelled propionate ester and $10 \mathrm{~g}$ of unlabelled ester. Breath $\mathrm{H}_{2}$ and ${ }^{13} \mathrm{CO}_{2}$ enrichment were collected over $24 \mathrm{~h}$ to investigate gut transit times. Breath $\mathrm{H}_{2}$ started to increase at $180 \mathrm{~min}$ and peaked at $240 \mathrm{~min}$. More than $80 \%$ of the ${ }^{13} \mathrm{C}$ recovered in breath appeared co-incident with and after breath $\mathrm{H}_{2}$ onset, suggesting delivery of the majority of the bound propionate to the colon.

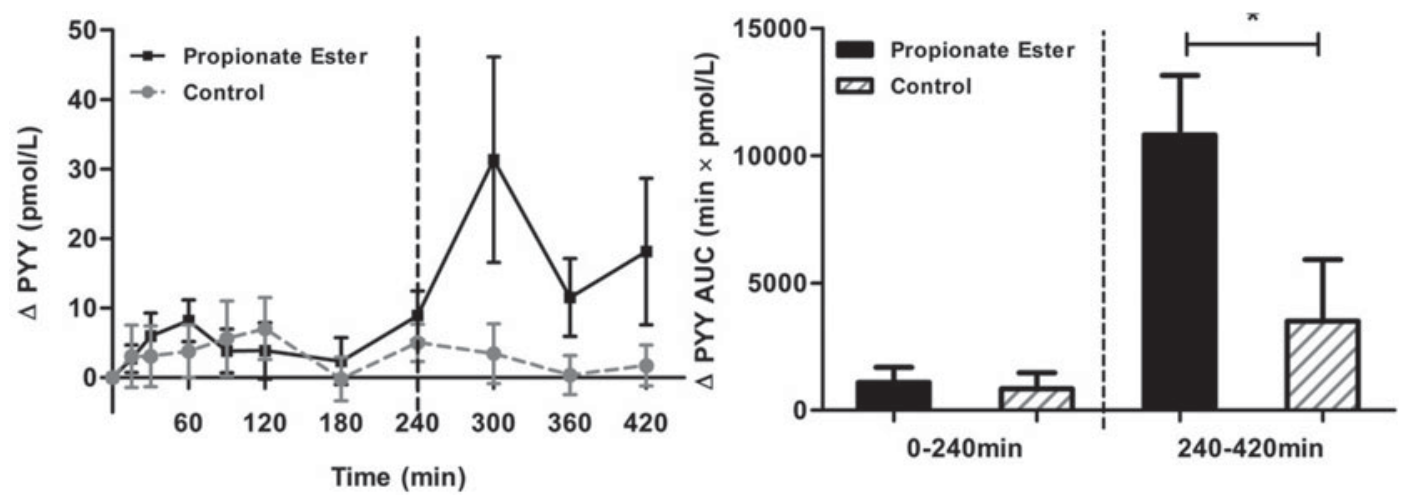

Plasma PYY levels after ingestion of propionate ester $v$ s. control. Dotted lines signify the time point after which $>80 \%$ propionate ester enters the colon. Data are presented as means \pm SEM, $* P<0.05$.

This propionate ester $(10 \mathrm{~g} / \mathrm{d})$ and an inulin control $(10 \mathrm{~g} / \mathrm{d})$ were then administered to 20 volunteers in a randomised crossover study to determine its effect on food intake and gut hormone concentrations. Ingestion of $10 \mathrm{~g}$ propionate ester significantly increased plasma PYY and GLP-1 $(P<0.05)$ and reduced ad libitum food intake $(1175 \pm 103$ kcal control vs. $1013 \pm 94$ kcal propionate ester; $p<0.01$ ).

In conclusion, these data suggest that an acute increase in colonic propionate can elevate plasma PYY and GLP-1 levels and reduce food intake in humans. Elevating propionate levels in the colon may therefore offer a potential strategy to protect against weight gain and the metabolic consequences of obesity.

This work was supported by the Biotechnology \& Biological Sciences Research Council (BB/H004971/1). All studies were conducted in accordance with the Declaration of Helsinki.

1. Tolhurst G, Heffron H, Lam YS, et al. (2011) Diabetes 61, 364-371.

2. Brown AJ, Goldsworthy SM, Barnes AA, et al. (2003) J Biol Chem 278, 11312-11319. 